\title{
DIVISIONS I and III / WORKING GROUP CARTOGRAPHIC COORDINATES AND ROTATIONAL ELEMENTS
}

\author{
CHAIR \\ VICE-CHAIR \\ PAST CHAIR \\ MEMBERS
}

\author{
Brent A. Archinal \\ vacant \\ P. Kenneth Seidelmann \\ Michael F. A'Hearn \\ Albert R. Conrad \\ Guy J. Consolmagno \\ Régis Courtin \\ Toshio Fukushima \\ Daniel Hestroffer \\ James L. Hilton \\ Gregory A. Neumann \\ Jürgen Oberst \\ Philip J. Stooke \\ David J. Tholen \\ Peter C. Thomas \\ Iwan P. Williams
}

\section{TRIENNIAL REPORT 2009-2012}

\section{Introduction}

As in the past, the primary activity of the IAU Working Group on Cartographic Coordinates and Rotational Elements has been to prepare and publish a triennial ("2009") report containing current recommendations for models for Solar System bodies (Archinal et al. (2011a)). The authors are B. A. Archinal, M. F. A'Hearn, E. Bowell, A. Conrad, G. J. Consolmagno, R. Courtin, T. Fukushima, D. Hestroffer, J. L. Hilton, G. A. Krasinsky, G. Neumann, J. Oberst, P. K. Seidelmann, P. Stooke, D. J. Tholen, P. C. Thomas, and I. P. Williams. An erratum to the "2006" and "2009" reports has also been published (Archinal et al. (2011b)). Below we briefly summarize the contents of the 2009 report, a plan to consider requests for new recommendations more often than every three years, three general recommendations by the WG to the planetary community, other WG activities, and plans for our next report.

\section{2009 Report Contents}

The 2009 WG report introduces improved values for the pole and rotation rate of Mercury (based on, but not precisely as recommended in Margot (2009)), returns the rotation rate of Jupiter to a previous value, introduces improved values for the rotation of five satellites of Saturn, and adds the equatorial radius of the Sun for comparison purposes. It also adds or updates size and shape information for the Earth, Mars' satellites Deimos and Phobos, the four Galilean satellites of Jupiter, and 22 satellites of Saturn. 
Pole, rotation, and size information has been added for the asteroids (21) Lutetia, (511) Davida, and (2867) Steins. Pole and rotation information has been added for (2) Pallas. Pole and rotation and mean radius information has been added for (1) Ceres. Pole information has been updated for (4) Vesta. The high precision realization for the pole and rotation rate of the Moon is updated to use the JPL DE 421 lunar ephemeris, but rotated (by small fixed angles) to represent the mean Earth/polar axis system. The WG has adopted the IAU Working Group for Planetary System Nomenclature (WGPSN) and the IAU Committee on Small Body Nomenclature (CSBN) definition of dwarf planets. As a result, Pluto and Charon now use the positive right handed coordinate system adopted for dwarf planets, minor planets, their satellites, and comets.

Upon request and to provide information more often than every three years (e.g., for use by missions and for new cartographic products) the WG announced it will consider providing limited updates to its recommendations on its (soon to be updated) web site (http://astrogeology.usgs.gov/Projects/WGCCRE). The tentative plan is to determine every 6 months whether time-critical updates are necessary and, if so, announce them on the site. We will also offer newly published and (preferably) peer-reviewed determinations related to Solar System coordinate systems. These postings do not remove the need for our triennial reports, in which we will continue to publish the majority of our recommendations. In our next report, we will consider the usefulness of these interim procedures and whether they should continue. Input for such updates (whether for WG consideration or information only) and comment on these procedures from the community is welcome.

For the first time, the WG also provided some general recommendations regarding current urgent needs relative to the development of planetary cartographic products. These include the following (paraphrased) recommendations: 1) The advantages of geodetically controlled cartographic products are many and well known, yet the current trend seems to be that such products are often not planned for or funded. It is strongly recommended that this trend be reversed and that such products be planned for and made as part of the normal mission operations and data analysis process. 2) The WG recommends that the Konopliv et al. (2006) Mars orientation model be updated or a similar model be developed that takes advantage of the substantial additional Mars data available since the time of their work, so that it can be adopted by the WG and operational Mars missions in the 2012 time frame. 3) The WG urges the planetary community to jointly address resolving the various determinations for the rotation of Jupiter and Saturn and to develop consensus determinations, such as was done in the past for Jupiter (Riddle and Warwick (1976)). The WG would like to hear feedback from the planetary community regarding these recommendations, e.g., actions planned or taken, the appropriateness of such recommendations, or even suggestions for further such general recommendations.

\section{Other Activities}

As part of other WG activities, a meeting of this group took place at the IAU General Assembly in Rio de Janeiro in 2009. Archinal was re-elected as chairperson and plans were discussed for the 2009 report. During the triennium members of the WG have also provided information and advice on planetary coordinate systems to various individuals, instrument teams, and missions. In particular, substantial advice was provided to MESSENGER mission representatives as to how best to update Mercury's coordinate system, to Cassini mission personnel on the updating of the coordinate system of various Saturnian satellites, to the Rosetta mission regarding the coordinate systems for (2867) 
Steins and (21) Lutetia, and to the Dawn mission regarding the coordinate system for (4) Vesta.

In the latter case, the Dawn mission proposed to establish a new prime meridian for Vesta in a substantially different location from the existing one, using primarily the argument that it was important to make it clear that the new high resolution data from Dawn were different from previously acquired datasets and therefore most appropriately presented in a different system. At the request of the Dawn mission, the WG formally voted on that type of system and voted to not concur with the proposed system. This was on the basis that it did not meet the long standing WG recommendation that "... once an observable feature at a defined longitude is chosen, the longitude definition origin should not change except under unusual circumstances..." (Archinal et al. (2011a)). The Dawn mission at the present time has stated that it plans to proceed with the new definition in spite of a lack of concurrence with IAU recommendations. The WG indicated that it will attempt to obtain feedback from the planetary community to see if having more than one prime meridian definition on a given body was now considered useful. We will take such feedback into account and consider for our next report whether to modify the WG recommendation on maintaining existing longitude systems that are tied to fixed features on a body.

The WG is also actively writing abstracts and making presentations at meetings to increase awareness of our activities. Examples have been presentations at the Lunar and Planetary Science Conference (Archinal (2011c)), the International Primitive Body Exploration Working Group meeting (Archinal (2011d)), the European Planetary Science Congress - Division of Planetary Sciences Joint Meeting (Archinal et al. (2011e)), and the International Society for Photogrammetry and Remote Sensing (ISPRS) Workshop on Geospatial Data Infrastructure (Archinal and Kirk (2011f)).

\title{
4. Current WG Plans
}

The Working Group is currently reformulating its membership and is starting to address changes needed for the next report. The WG currently anticipates updates or new values in several areas including a) the use of an improved lunar ephemeris to define the Moon's orientation, either from JPL or others; b) updates for the orientation of Mars, Jupiter, and Saturn (as already noted); and c) updates due to new results from on-going missions (Mercury, Saturnian satellites, (4) Vesta, (21) Lutetia) and Earth-based observations (various asteroids). The WG also will look into establishing or re-establishing links to other organizations, such as the International Association of Geodesy and the ISPRS. On a "reasonable effort" basis, it will continue to provide assistance on coordinate system and mapping issues to members of the international planetary science community (space agencies, missions, instrument teams, product developers, etc.).

A draft of the next report will be presented for discussion and completion at the Working Group meeting at the IAU General Assembly in Beijing in 2012.

\author{
Brent A. Archinal \\ chair of Working Group
}

\section{References}

Archinal, B. A. et al. 2011a, Cel. MEDA 109, 101-135, doi 10.1007/s10569-010-9320-4 Archinal, B. A. et al. 2011b, Cel. MEDA 110, 401-403, doi 10.1007/s10569-011-9362-2 Archinal, B. A. 2011c, LPS XLII, abs. 2715 
Archinal, B. 2011d, IPEWG 2011, http://ipewg.caltech.edu/

Archinal, B. et al. 2011e, EPSC-DPS Joint Meeting 2011, abs. 1553

Archinal, B. A. \& Kirk, R. L. 2011f, ISPRS Workshop, http://isprs-wg41.nsdi.gov.cn/

Konopliv, A. S. et al. 2006, Icarus 182, 23-50

Margot, J.-L. 2009, Cel. MEDA 105, 329-336, doi 10.1007/s10569-009-9234-1

Riddle, A. C. \& Warwick, J. W. 1976, Icarus 27, 457-459 